\title{
Design and test of HiSGR: a novel GNSS/INS ultra tight coupled receiver
}

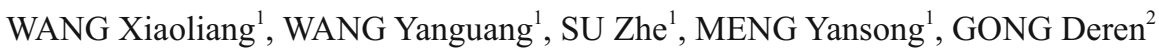 \\ 1. Institute of Satellite Navigation and Intra-Satellite-Link Technology, Academy of Space Electrical Information Technology, Xi'an \\ 710100, China \\ 2. School of Aeronautics and Astronautics, Shanghai Jiao Tong University, Shanghai 200230, China
}

\begin{abstract}
With the support of the national nature science foundation, the Academy of Space Electronic Information Technology is developing a novel compact spaceborne GNSS receiver, referred to as the HiSGR (High Sensitivity GNSS Receiver). This receiver can operate effectively in the full range of Earth orbiting missions, from LEO (Low Earth Orbit) to geostationary and beyond. Improved signal detection algorithms are used in the signal process section of the HiSGR and an inertial sensor is used for GNSS/INS ultra tight coupled design, which makes the acquisition process fast and provides improved tracking performance for weaker GPS signals in the presence of high dynamics. Extensive tests are performed using the HiSGR to demonstrate the good performance of some crucial specifications, by employing a real GNSS signal received in an open field and through hardwarein-the-loop simulation. Receiver performance is demonstrated for LEO and GEO scenarios. A ground vehicle running test is performed for demonstration of fast acquisition and reacquisition capabilities under conditions of signal loss. The HiSGR showed good performance and it was stable during the simulations and tests, which proved its capability for future space applications.
\end{abstract}

Key words: high sensitivity, GNSS/INS, ultra tight coupled, spaceborne receiver.

Citation: WANG X L, WANG Y G, SU Z, et al. Design and test of HiSGR: a novel GNSS/INS ultra tight coupled receiver[J].

Journal of communications and information networks, 2016, 1(3): 67-76.

\section{Introduction}

In recent years, the use of GNSS receivers for space missions has become very common. Some major applications include ${ }^{[1]}$ the following.

1) Real-time POD (Precise Orbit Determination): The receiver provides accurate three-dimensional position and velocity information onboard for spacecraft, thereby improving spacecraft autonomy and simplifying the ground tracking and ranging segment. Moreover, the receiver can provide accurate reference time that is synchronized to UTC with an accuracy of better than $1 \mu \mathrm{s}$, which is particularly useful for telecommunications and Earth observation missions where accuracy and latency are critical.

2) Attitude determination: Using three or four GNSS antennas connected to the receiver that performs GNSS signal carrier difference measurements, the attitude information of the spacecraft can be obtained in real time.

3) Raw measurement provider: The receiver can provide all types of raw GPS measurements that

Manuscript received Sept. 14, 2016; accepted Sept. 25, 2016

This work is supported by the National Natural Science Foundation of China (No.91438107). 
can be either transmitted to another spacecraft for relative navigation, or downloaded to the ground for precise post-processing. Applications of these types of space missions include satellite remote sensing, ocean altimeters, and synthetic aperture radar mappers. While these missions may not require real-time accuracy, the ability to achieve decimeter-level accuracy onboard would allow for completed science products in near real time that are ready for immediate analysis, thus eliminating extra analysis cost and time.

Since there is a high demand for space applications of GNSS receivers, several organizations have undertaken efforts to develop spaceborne GNSS receivers for LEO to lunar missions in the last two decades. Some well-known products include the BlackJack receiver (Turbo Rogue Space Receiver, TRSR-2) $)^{[2,3]}$ and a follow-on version developed by NASA's Jet Propulsion Laboratory and Broad Reach Engineering ${ }^{[4]}$, a commercial-off-the-shelf dualfrequency receiver (OEM4-G2L) from NovAtel Inc. for space applications, and Septentrio's PolaRx2 receiver for LEO missions ${ }^{[5,6]}$.

ASEIT is currently developing a novel compact spaceborne GNSS receiver, referred to as "HiSGR," for future space applications, with the support of National Nature Science Foundation. The receiver is shown in Fig.1. This paper introduces HiSGR

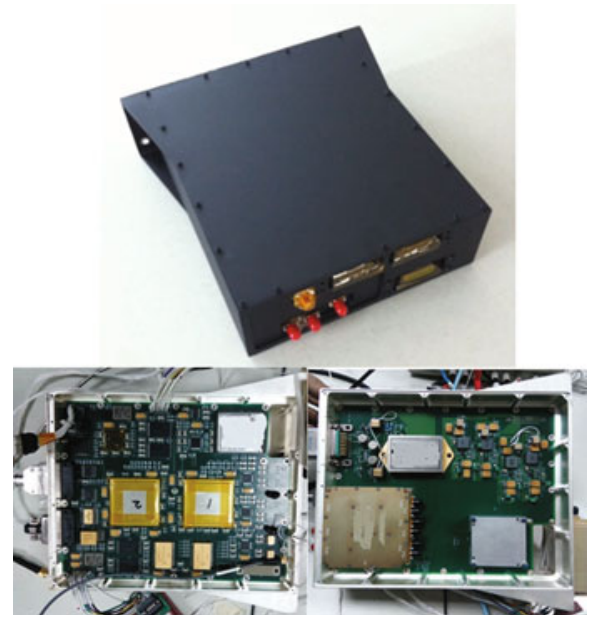

Figure 1 HiSGR receiver and internal components receiver in detail. The rest of the paper is organized as follows: section two introduces the hardware and software architectures of the HiSGR receiver. The results of the tests carried out using HIL (HardwareIn-the-Loop) simulation and real signals are given in section three.

\section{Receiver architecture}

\subsection{Hardware}

As shown in Fig.2, all receiver components are laid out on two printed circuit boards. The bottom layer includes the following:

1) A high-sensitivity front end with a LNA (Low Noise Amplifier) that provides a down-converted GNSS signal. 2) A DC-DC converter power supply module that provides the necessary second voltages to the other components from the primary $28 \mathrm{~V} \mathrm{DC}$ power bus. 3) A high-stability frequency reference. 4) An inertial measurements sensor. All the information is transferred to the top layer for post processing. The output navigation solutions and necessary raw measurements are sent to a remote-terminal command and telemetry interface using the MIL-STD-1553B communication bus, and finally sent to a spacecraft GNC system through an internal connector.

Two GNSS receiver antennas are used, one zenith pointing and one nadir pointing. This is because high altitude spacecraft perform signal acquisition and tracking of GPS satellites in the down-looking direction. The two antennas can be switched on and off automatically in the front-end device, according to the altitude of the receiver ${ }^{[7]}$, and intermittent frequency signals are obtained. The current version of the HiSGR is a GPS L1/L2 dual-frequency receiver that is capable of receiving $\mathrm{L} 1 \mathrm{C} / \mathrm{A}, \mathrm{L} 2 \mathrm{C}$, and $\mathrm{L} 2 \mathrm{P}$ codes. Receiving BeiDou B1 and B3 signals will be realized in the near future. 


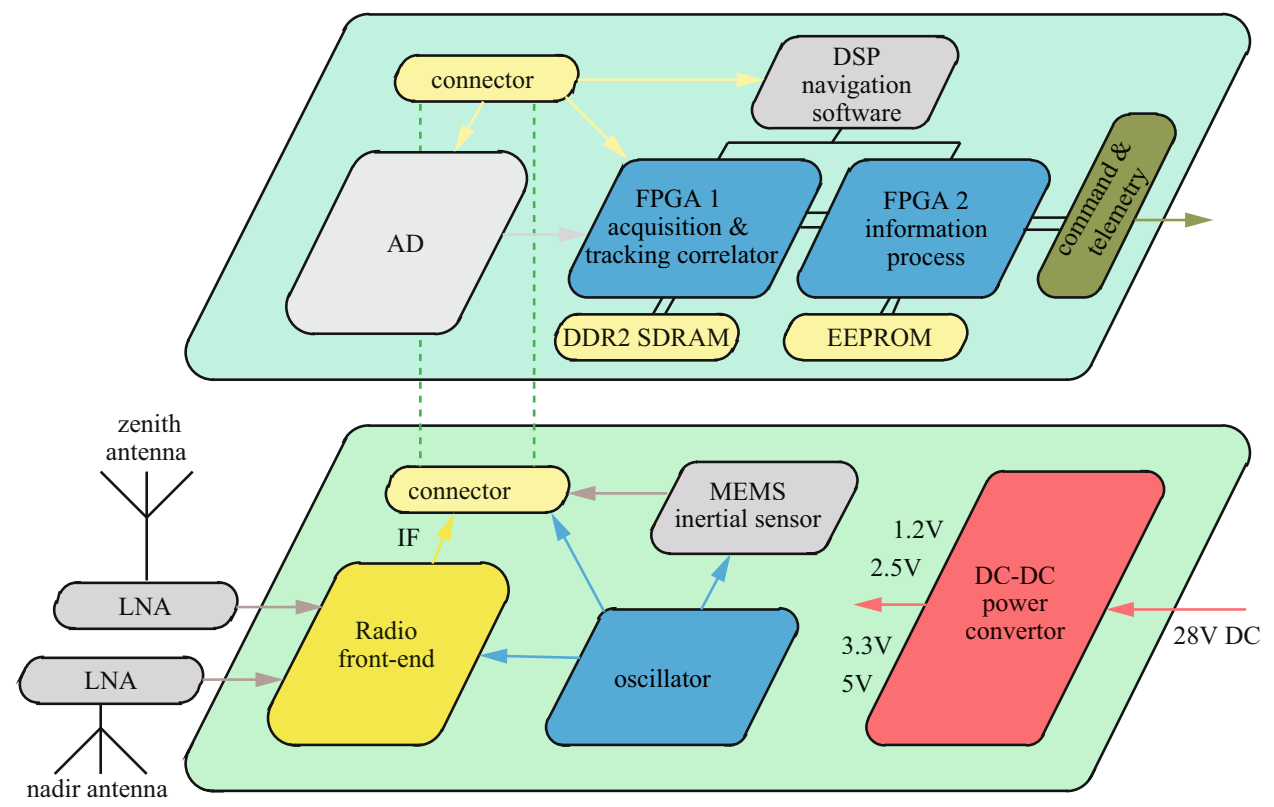

Figure 2 HiSGR receiver hardware structure

Compared to ordinary temperature compensated crystal oscillators, the oscillator used in the HiSGR provides good stability performance for missions involving poor GNSS visibility in high Earth orbit. For improving receiver sensitivity in weak signal acquisition, a MEMS (Micro Electro Mechanical System) based IMU (Inertial Measurement Unit) component from ADI Inc. has been used in the HiSGR.

FPGA1 in the top layer is an acquisition \& tracking correlator FPGA, controlled using an internal microprocessor, which is used for fast weak-signal acquisition and tracking. This device performs the most computationally demanding task that requires a search across a three-dimensional space of unknown time delay, Doppler shift, and the pseudo random noise number of the satellite, that are critical for the sensitivity of traditional receiver design. Improved signal detection algorithms have been used in the HiSGR; these algorithms speed up the acquisition process and provide improved performance for weaker GPS signals in the presence of high dynamics.
Typically, tracking of signals down to $25 \mathrm{~dB}-\mathrm{Hz}$ is possible using standard GPS methods ${ }^{[8]}$, while acquisition is limited to approximately $35 \mathrm{~dB}-\mathrm{Hz}$. Special implementations of an acquisition scheme are used for the HiSGR. First, the satellite selection logic from the software section can predict the GNSS satellite passes and the coarse Doppler shift for any altitude and antenna orientation. Second, an ultra tight coupled GNSS/inertial Kalman filter is used during dynamic maneuvers and in challenging signal environments (launch, orbit transitions, and reentry), and it aids in fast signal acquisition or reacquisition.

The tracking correlator implements the code delay locked loop and carrier tracking functions on each of the channels, with data output by the acquisition section. The data are stored in a fast DDR2 SDRAM that is connected to FPGA1. By optimal design of the code and carrier tracking loops for the expected signal levels and dynamics in space, it is possible to increase the sensitivity of the receiver for tracking weaker GPS signals that are at or just below the tracking threshold of typical receivers. Conventional GPS acquisition 
$\&$ tracking algorithms use $1 \mathrm{~ms}$ accumulated $I$ and $Q$ values, as this is convenient for detecting the data bit transitions. The longer the accumulation time, the lower the satellite $C / N_{0}$ that can be detected. Detection thresholds are typically set at approximately $95 \%$ probability. This is equivalent to a post-accumulation signal/noise level of approximately $8 \mathrm{~dB}$. Two methods are used for improving weak signal tracking performance in the HiSGR. The first is using longer non-coherent accumulations, and the second is using an adaptive tracking loop filter. The first method uses $20 \mathrm{~ms}$ correlations for tracking weak signals, and 50 non-coherent accumulations over $1 \mathrm{~s}$, using $20 \mathrm{~ms}$ coherent data. The $C / N_{0}$ detection threshold that is achieved in tests is approximately $15 \mathrm{~dB}-\mathrm{Hz}$ or even lower. The adaptive tracking loop filter is also used for reliable tracking and data demodulation of the $C / A$ signal, by estimating the best gain settings of tracking loops in real time ${ }^{[9]}$. However, the filter method is computationally intensive, which makes it unsuitable for routine use, especially in strong signal scenarios.

FPGA2 is used for inertial measurements, and aided almanac information collection from the MEMS sensor in the bottom layer and the attached EEPROM. It also controls the navigation solutions output from the DSP and handles command and telemetry messages thought MIL-STD-1553B interface ports.

\subsection{Algorithm}

The structure of the receiver algorithm is shown in Fig.3. The inertial computation section feeds the residual measurements and associated accuracies from all satellites into an ultra tight coupled algorithm, in parallel. The resulting vehicle position and velocity estimates are then projected along the line of sight of each satellite to close the vectortracking loop.

The inertial compute section estimates vehicle

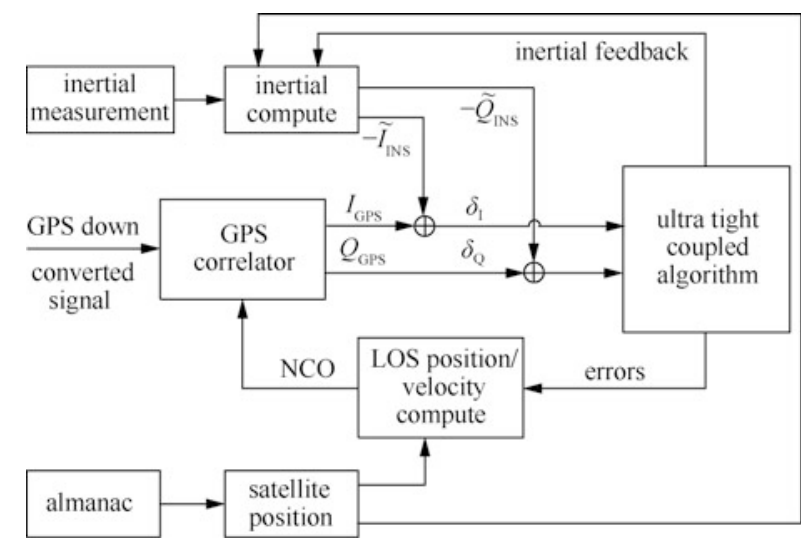

Figure 3 HiSGR algorithm structure

position, velocity, attitude, clock, and IMU error parameters at a slow rate (e.g., one second intervals). The IMU vehicle angular rate and specific force measurements are compensated for coning and sculling, and adjusted using the estimated error parameters. The IMU measurements are then forward integrated from the inertial computation to obtain the vehicle position, velocity, and attitude estimates at a fast rate (e.g., $200 \mathrm{~Hz}$ ).

These estimates are projected along the line of sight of each satellite to obtain range and range rate estimates, which are used to drive the code and carrier NCOs. The carrier NCO output is mixed with the GPS signal phase, resulting in phase error. The code NCO drives the code generator. The generator's output is correlated with the incoming GPS code, resulting in a correlation magnitude between zero and one.

\section{Test and simulations}

Extensive tests were performed using the HiSGR to demonstrate the good performance of some crucial specifications, by employing HIL simulation and a real GNSS signal received from a vehicle moving in an open field and underground.

The following subsections describe the HIL simulation configuration, general HiSGR receiver 
performance, data processing, and preliminary results.

\subsection{HIL simulation for LEO}

HIL testing is useful for evaluating the actual performance of the receiver under different conditions including dynamics, signal levels, and error sources on orbit. In this test, the RF input of the receiver is connected to a GPS simulator rather than to a real antenna. The specified trajectory and attitude can be provided from the simulator. Aspects of the receiver performance can be evaluated repeatedly, which is impossible using terrestrial static tests.

ASEIT has recently purchased a state-of-theart Spirent GNSS simulator (GSS9000, shown in Fig.4, with HiSGR connection), which is a multiple constellation/frequency GNSS simulator (GPS/ Galileo/BeiDou/GLONASS/SBAS/QZSS). It has up to 16 parallel channels per carrier, through each RF output. This simulator allows users to virtually control all properties of the simulated GNSS signal, and modeled error sources.

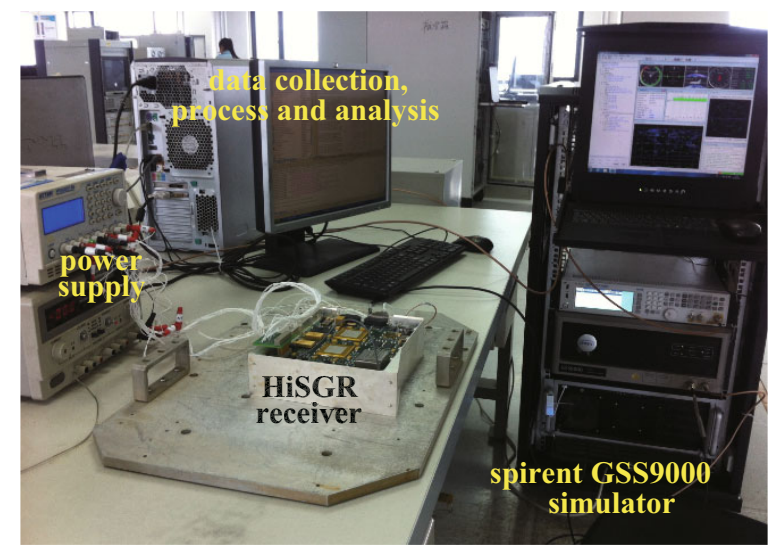

Figure 4 HiSGR receiver under HIL test

The LEO scenario of CHAMP (Challenging Minisatellite Payload) is used in this simulation, the orbit elements for which are given in Tab.1. These elements demonstrate a typical Earth gravity observation mission in low altitude.
Table 1 Orbit elements of CHAMP

\begin{tabular}{cc}
\hline items & data \\
\hline eccentricity & 0.00398 \\
inclination & $87.272^{\circ}$ \\
perigee altitude & $429 \mathrm{~km}$ \\
apogee altitude & $476 \mathrm{~km}$ \\
longitude of ascending node & $150.089^{\circ}$ \\
argument of perigee & $323.038^{\circ}$ \\
\hline
\end{tabular}

The receiver antenna is configured to be fixed to the center of mass of the spacecraft, and is oriented in the zenith direction. The receiving antenna gain patterns can be configured manually using an antenna attenuation pattern editor in the GSS9000 simulator; the default gain patterns are used in this simulation (default_v1-0.ant_pat file in setting GUI).

Only the GPS constellation is used in the test. The signal levels in the simulator are set at a default value of $-130 \mathrm{dBm}$, which complies with the minimum guaranteed signal strength from GPS ICD$200^{[10,11]}$.

The simulation time is set from 14:15:00 to 20:15:00 08/Apr/2016 UTC, with a sampling step of $1 \mathrm{~s}$. The GPS reference orbits are based on a precise ephemeris, which is downloaded as a version 3.01 RINEX file from the IGS website (http://igscb.jpl. nasa.gov/), and it makes the simulated GPS orbits and clock parameters closer to those of the actual GPS constellation. RTPODs-LEO (Real time precise orbit determinations-LEO) software is burned into the hardware components; it is a GNSS only version without an inertial assistant. The signal acquisition and tracking algorithm uses $1 \mathrm{~ms}$ correlations for this test.

The top sky plot obtained from the simulation is shown in Fig.5. For conciseness, the trajectory of only the GPS satellite with PRN 15 is shown (red lines in Fig.5). The initial position of all visible satellites is also shown (blue squares). It is interesting to note that the receiving elevation angle that is achieved during 


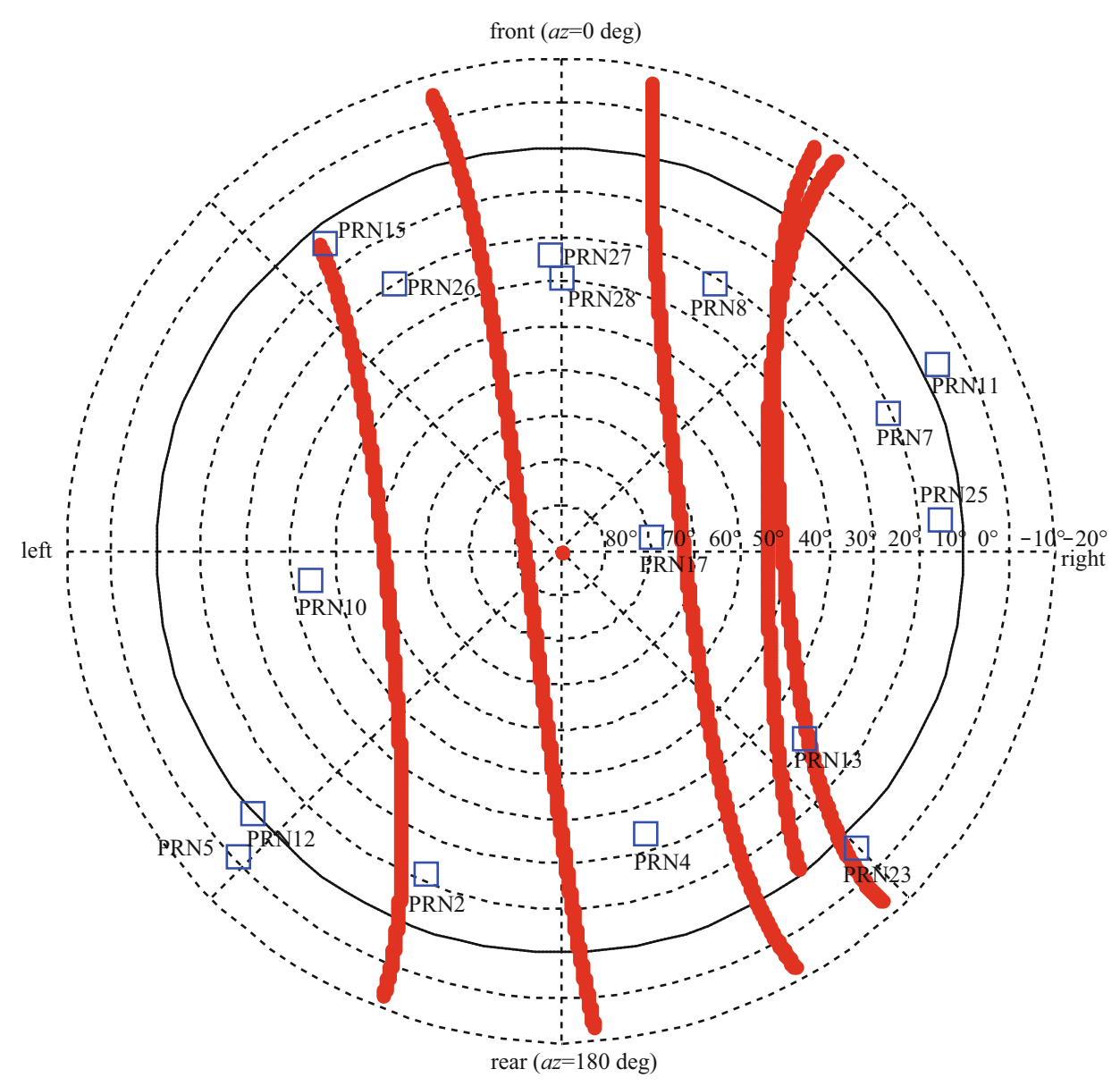

Figure 5 Top sky plot analysis

the simulation is almost $-20^{\circ}$. This is because of the following two reasons: 1) The receiving antenna setting file (default_v1-0.ant_pat) in the simulator is sphere equal attenuation in all directions, and 2) the Earth tangent setting in the GSS9000 simulator GUI is just above the Earth obscuration angle, which ensures signal reception at a low elevation angle, as shown in Fig.6.

The HiSGR showed excellent performance throughout the simulation, and provided stable signal processing capability in LEO scenarios. Fig.7 shows the PDOP values and the histogram of satellite geometry visible and satellites get tracked data from the output of the RTPODs-LEO software. The POD performance after filter convergence is shown in Fig.8 (using $15 \mathrm{~s}$ sample interval points for conciseness).

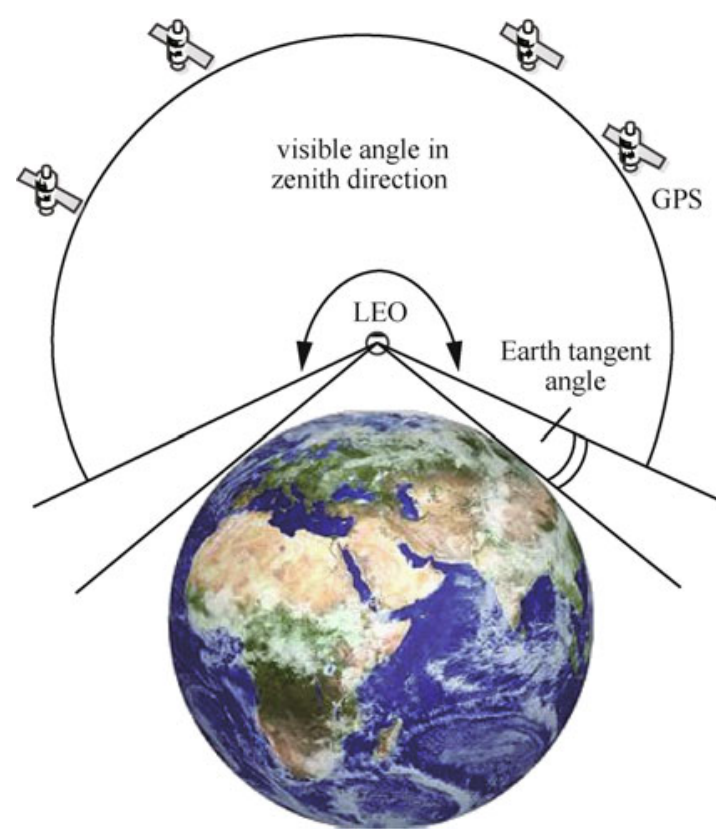

Figure 6 Earth tangent setting in simulator 
The position errors in the radial/ in-track/ cross-track directions are approximately a few meters in realtime processes, which is suitable for most missions in LEO with real-time requirements.

(a)

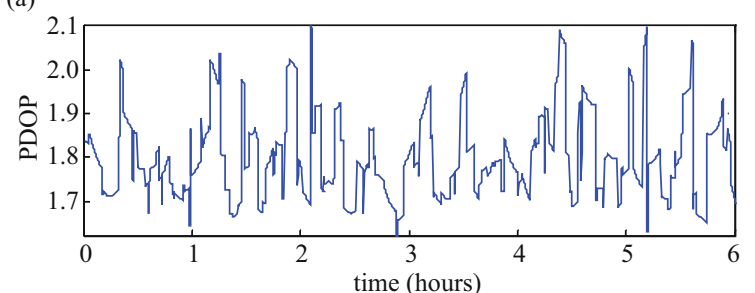

(b)

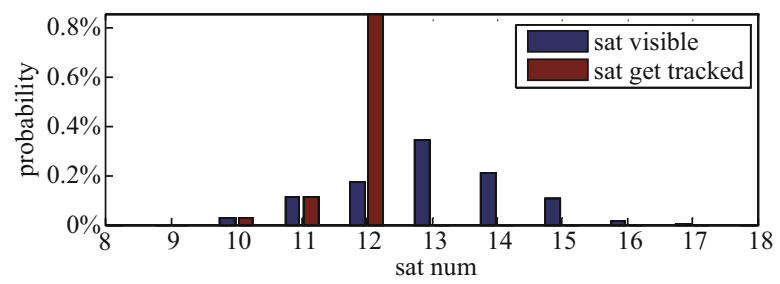

Figure 7 the PDOP values and the histogram of satellite geometry visible and satellites get tracked data: (a) PDOP; (b) GPS visible analysis

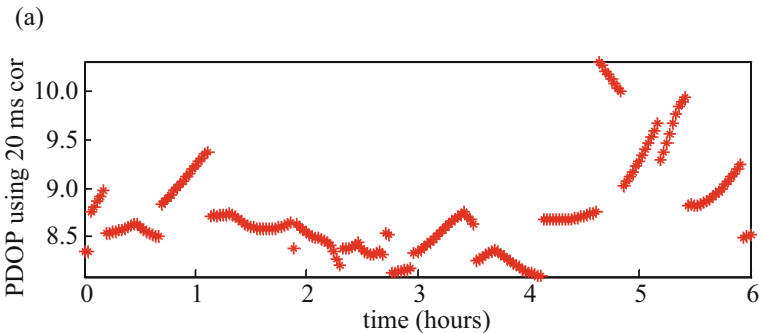

(b)

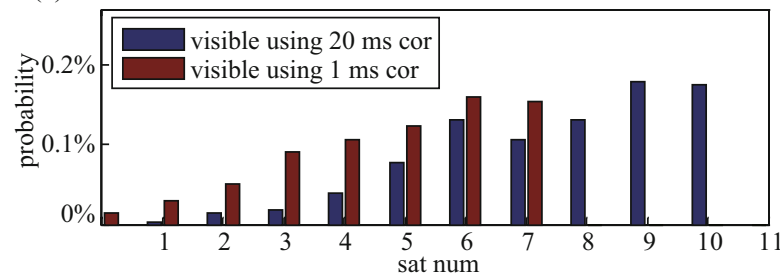

Figure 8 histograms of the percentage of the time vs. the number of satellites: (a) PDOP; (b) visible analysis

\subsection{GEO simulation}

This section presents some results of the tests that were conducted for a geostationary orbit, in which the
HiSGR receiver operated for 6 hours. The scenario assumes a single nadir-pointing and high gain receiving antenna with a boresight angle of $\pm 45^{\circ}$ and a gain of $5 \mathrm{~dB}$.

The bottom of Fig. 8 shows histograms of the percentage of the time vs. the number of satellites that were tracked simultaneously, which demonstrated the suitability of the HiSGR for real applications. The results indicate that four or more satellites can be tracked simultaneously for more than $90 \%$ of the time. The receiver performed reasonably well at tracking the GPS signals that were above its nominal tracking threshold.

Like the top sky plot shown in Fig.5, Fig.9 provides a way to visualize the GPS constellation from the perspective of the GEO spacecraft, un-der the $1 \mathrm{~ms}$ correlations condition. A typical GPS satellite can be acquired and lost as it passes behind Earth and through the GPS side lobes to the main lobe.

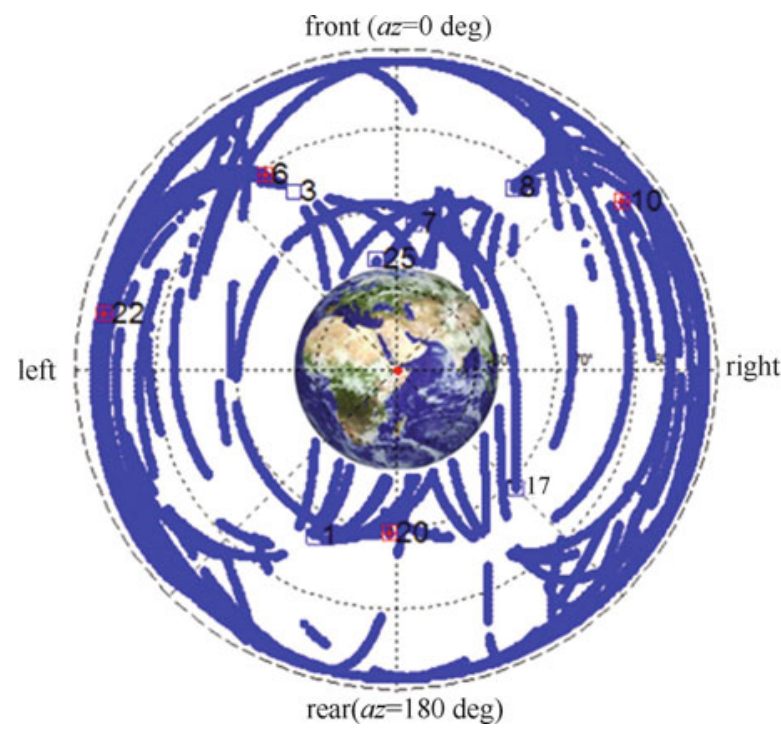

Figure 9 Nadir sky plot analysis for GEO

\subsection{Vehicle test on ground}

This section briefly discusses the results of the tests that were carried out using a vehicle moving on an open field and underground, as shown in Fig.10. 


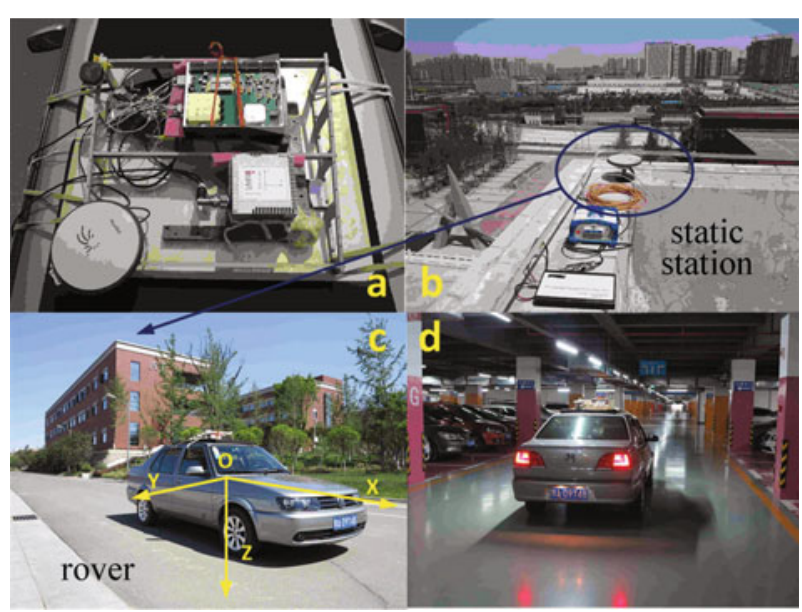

Figure 10 Vehicle tests on open field and underground: (a) HiSGR on the moving vehicle; (b) static station of Novatel receiver; (c) coordinate of vehicle; (d) vehicle moving underground

An ultra tight coupled software algorithm is used for weak signal acquisition and tracking under different conditions; it is currently is under development and has limited functions. However, the signal processing is carried out using inertial assistants, and the position is computed offline using sampled data during the test. Fig.10(a) shows the assembling of the HiSGR on the platform with an integrated navigation system (SPAN@, NovAtel), which is used for the comparison and evaluation of the final results. Fig.10(b) and (c) show the basic instrument configurations during the test, and Fig.10(d) shows the vehicle moving underground, where the aim is to assess the receiver performance when no visible satellites are available in space and to achieve fast signal reacquisition.

The receiver achieved fast signal reacquisition when it was outside the underground garage, and the HiSGR performance was stable during the test on the open field, as shown in Fig.11. The left and right of Fig.11 show the "real" trajectory obtained using the SPAN system and the HiSGR. The position error is less than 10 meters during the test. Currently, only a few primary results are obtained in the ground test. More information about the HiSGR test results will be provided in the authors' subsequent publications.

(a)
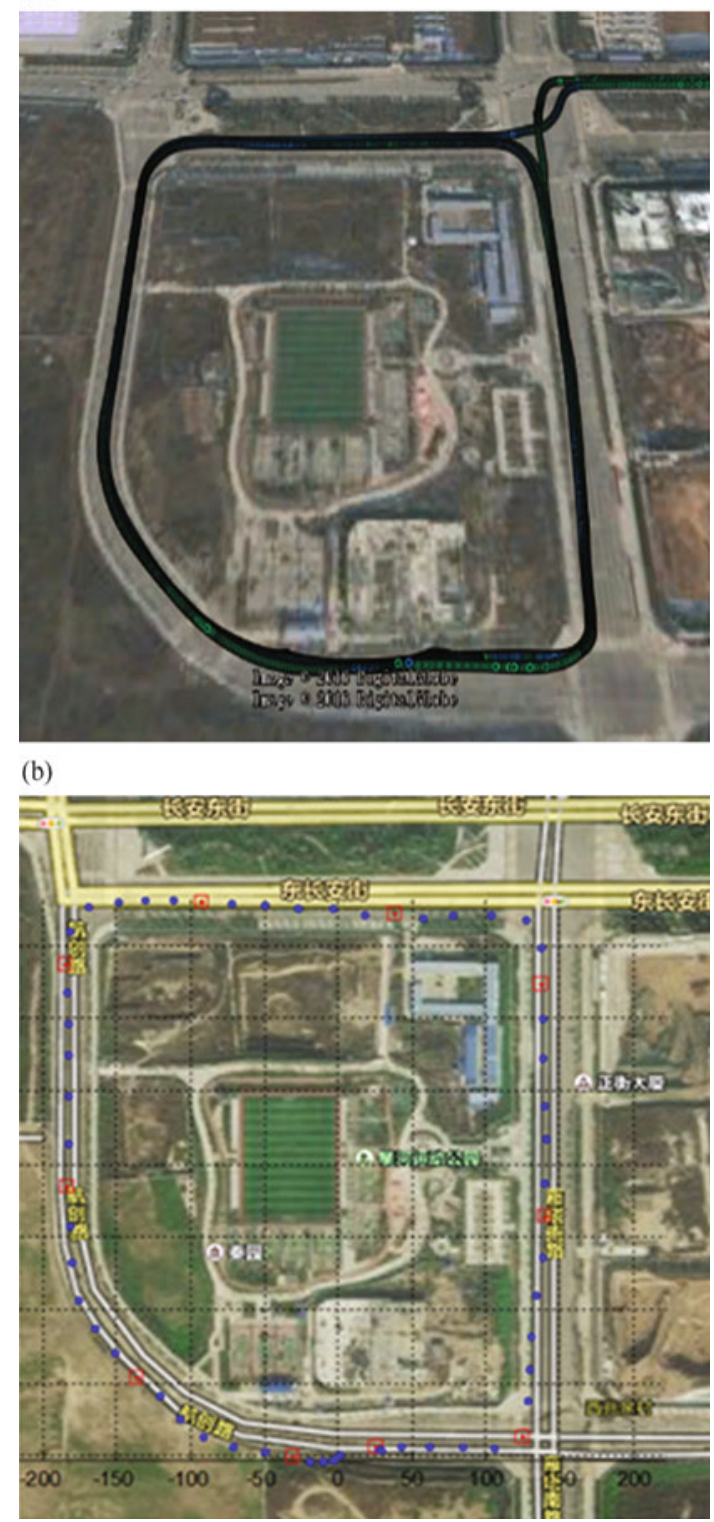

Figure 11 Vehicle tests on open field: (a) ground trajectory from Novatel receiver; (b) ground trajectory from HiSGR

\section{Conclusion}

This paper provided a detailed introduction of the design and development of a novel compact spaceborne GNSS receiver (HiSGR), which was carried out by ASEIT. 
The HiSGR is a space qualified multifunctional GNSS receiver that can operate effectively in the full range of Earth orbiting missions, from LEO to geostationary and beyond. Improved signal detection algorithms are used in the signal process section of the HiSGR. This makes the acquisition process fast, and provides improved tracking performance for weaker GPS signals. Extensive tests were performed using the HiSGR, by employing HIL simulation and a real GNSS signal received from a vehicle moving in an open field and underground. The real-time POD positioning error is approximately a few meters in LEO, and less than $50 \mathrm{~m}$ in GEO. The simulation results showed the good performance of the HiSGR, which makes it suitable for future space applications.

\section{References}

[1] BAUER F H, HARTMAN K, LIGHTSEY E G. Spaceborne GPS: current status and future visions $[\mathrm{C}] /$ Proceedings of the Institute of Navigation GPS 98 Conference, Nashville, USA, 1998: 1493-1508.

[2] MONTENBRUCK O, KROES R. In-flight performance analysis of the CHAMP BlackJack GPS receiver[J]. GPS solutions , 2003, 7(2): 74-86.

[3] REICHERT A, MEEHAN T, MUNSON T. Toward decimeter-level real-time orbit determination: a demonstration using the SAC-C and CHAMP spacecraft[C]//Proceedings of International Technical Meeting of the Satellite Division of the Institute of Navigation, Portland, USA, 2002: 1996-2003.

[4] WU B H, CHU V, CHEN P, et al. FORMOSAT-3/COSMIC science mission update[J]. GPS solutions, 2005, 9(2): 111-121.

[5] LANGLEY R B, MONTENBRUCK O, MARKGRAF M, et al. Qualification of a commercial dual-frequency GPS receiver for the e-POP platform onboard the Canadian CASSIOPE spacecraft[C]// Second ESA workshop on satellite navigation user equipment technologies, NAVITEC, Noordwijk, Netherlands, 2004: 8-10.

[6] LEYSSENS J, MARKGRAF M. Evaluation of a commercial-offthe-shelf dual-frequency GPS receiver for use on LEO satellites[C]// Proceedings of International Technical Meeting of the Satellite Division of the Institute of Navigation, Long Beach, USA, 2005.

[7] WANG X L, LI L L, HAN X Y, et al. Analysis of GNSS signals with application to lunar navigation[C]// Proceedings of China Satellite Navigation Conference, Xi'an, China, 2015, I(18): 1-8.

[8] DIERENDONCK A V. Global positioning system: theory and applications. reston[M]. VA: Amer. Inst. of Aeronaut. and Astronaut., 1996: 329-407.

[9] WANG Y G, TIAN J, LECLÈRE J, et al. An efficient timefrequency algorithm for weak signal acquisition of modernized GNSS signals[C]//Ion Gnss, 2014, 57(6): 715-722.

[10] ANON. GPS Interface control document[R]. ICD-GPS-200, Revision C, U.S. Air Force, 1993.

[11] RYCROFT M J. Understanding GPS: Principles and applications[M]. London: Artech House Publishers, 1996.

\section{About the authors}

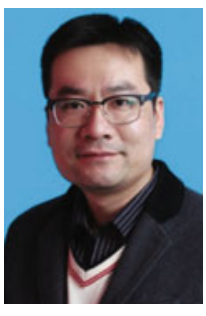

WANG Xiaoliang [corresponding author] was born in 1981. He received a Ph.D. degree major in navigation, guidance and control in Institute of Aeronautics and Astronautics, Shanghai Jiao Tong University in 2011. Received M.S. and B.S. degrees in system control engineering from Northwestern Polytechnic University in 2006 and 2003 respectively, Xi'an, China. He has been with Institute of Satellite Navigation and Intra-Satellite-Link Technology, Academy of Space Electronic Information Technology, Xi'an, China, since 2011. He is a recognized expert in the field of GNSS applications in space and currently is the principle leader of HiSGR project. His research interests include design and evaluation of spaceborne GNSS receiver for multiple missions, weak signal acquisition \& tracking, ultra-tight coupled GNSS/Inertial technology in space, autonomous real-time orbit determination onboard, and nonlinear Kalman Filter technology. (Email: xlwang12321@gmail.com, xlwang12321@foxmail.com)

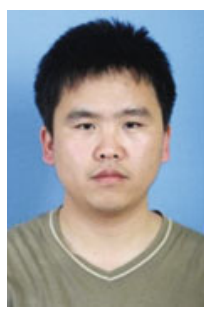

WANG Yanguang received a master degree in electronics and signal processing from the Xidian University, Xi'an, China, in 2007. He is currently with Institute of Satellite Navigation and IntraSatellite-Link Technology, Academy of Space Electronic Information Technology. He has been working on the field of GNSS applications in space for years, research interests including acquisition and tracking for weak GNSS signal, hardware design and development of high sensitivity spaceborne receiver. 


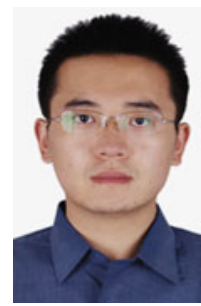

SU Zhe received a Ph.D. degree in circuits and system from Institute of Electronic Engineer, Xidian University, Xi'an, China, in 2011. He is currently with Institute of Satellite Navigation and Intra-Satellite-Link Technology, Academy of Space Electronic Information Technology. His research interest include autonomous navigation using X-ray pulse star technology, design and test of new generation GNSS signals and onboard payload.

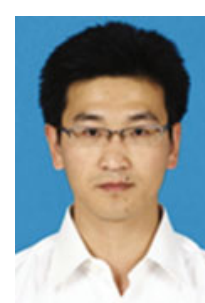

MENG Yansong received M.S. degree from Academy of Space Electronic Information Technology, Xi'an, China, and worked there since 2005 . He is now a $\mathrm{Ph} . \mathrm{D}$. candidate of Beijing Institute of Technology. His research interests include onboard payload design and analysis of navigation satellites, intrasatellite-link and precise time frequency transfer technology.

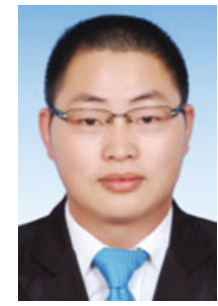

GONG Deren received a Ph.D. degree major in navigation, guidance and control in School of Aeronautics and Astronautics, Shanghai Jiao Tong University in 2012. Received M.S. and B.S. degrees from Xi'an Jiaotong University in 2006, 2003, respectively, Xi'an, China. He has been worked as a postdoctoral research fellow in Shanghai Jiao Tong University during 2012-2014, and currently work there as an associate research fellow. His research interests including guidance, navigation and control of spacecraft orbit and attitude, Precise modeling of spacecraft dynamic and kinematics, nonlinear estimation and control of spacecraft formation flying and rendezvous $\&$ docking process, HIL (Hardware In the Loop) simulation for massive aerospace system. (Email: drgong@sjtu.edu.cn) 